\title{
Osteonecrosis of the jaw in a patient taking once-yearly infusion of zoledronic acid for osteopenia
}

\author{
Takako Imai Tanaka, Charles Donald Taylor \\ ${ }^{1}$ Department of Biomedical and Diagnostic Sciences, University of Detroit Mercy School of Dentistry, Detroit, USA; \\ *Corresponding Author: tanakata@udmercy.edu \\ ${ }^{2}$ Department of Patient Management, University of Detroit Mercy School of Dentistry, Detroit, USA; \\ taylorcd@udmercy.edu
}

Received 14 September 2013; revised 10 October 2013; accepted 7 November 2013

Copyright (c) 2013 Takako Imai Tanaka, Charles Donald Taylor. This is an open access article distributed under the Creative Commons Attribution License, which permits unrestricted use, distribution, and reproduction in any medium, provided the original work is properly cited. In accordance of the Creative Commons Attribution License all Copyrights ( 2013 are reserved for SCIRP and the owner of the intellectual property Takako Imai Tanaka, Charles Donald Taylor. All Copyright (c) 2013 are guarded by law and by SCIRP as a guardian.

\section{ABSTRACT}

Osteonecrosis of the jaw (ONJ) is an adverse effect of nitrogen-containing bisphosphonates. Advancing age, intravenous administration of zoledronic acid (ZOL), history of dento-alveolar surgery, and concomitant systemic diseases such as diabetes are known as risk factors for developing ONJ. However, despite numerous studies, the exact pathophysiology remains unclear and management strategies are largely anecdotal. Once-yearly intravenously administered $5 \mathrm{mg} \mathrm{ZOL}$ was approved by the US Food and Drug Administration in 2007 for the treatment of osteoporosis and its efficacy with 3 year-regimen had been recently proven in preventing new clinical fracture. Although occurrences of ONJ have been reported to be rare with this drug administration, available data is very limited and long-term outcomes are lacking. We present a case of ONJ identified in an osteopenic patient with an intermittent but long standing sore mouth related to exposed mandibular bone. Once-yearly infusion of zoledronic acid used in the treatment of osteopenia may contribute to the spontaneous development of ONJ, especially in those presenting with multiple comorbidity factors. This report suggests the importance of health care professionals keeping abreast of new developments in this area and providing appropriate information to their patients.
Keywords: Osteopenia; Osteoporosis;

Bisphosphonates; Osteonecrosis; Letrozole;

Once-Yearly Zoledronic Acid; ONJ; ARONJ; BRONJ

\section{INTRODUCTION}

Osteonecrosis of the jaw (ONJ) was first reported as non-healing tooth extraction sites in cancer patients taking nitrogen-containing bisphosphonates (NBPs) in 2003 [1]. Emerging cases of ONJ related to a wider variety of medication use in osteoporosis such as Denosmub have led to the introduction of new terminology "antiresorptive agent-induced ONJ, "ARONJ" to replace bisphosphonate-related ONJ, "BRONJ". Incidence of ARONJ is estimated as small as $0.1 \%$ based on current available evidence [2]. The risk factors for developing ARONJ include advancing age, intravenous administration of NBPs (especially zoledronic acid: ZOL), history of dentoalveolar surgery, and concomitant systemic diseases such as diabetes. Despite numerous studies, the exact pathophysiology remains unclear and management strategies are largely anecdotal.

Once-yearly intravenously administered $5 \mathrm{mg}$ ZOL was approved by the US Food and Drug Administration in August 2007 for the treatment of osteoporosis. Its efficacy with 3 year-regimen has been proven significant in preventing new clinical fracture [3]. Although occurrences of ONJ have been reported to be rare [4], available data are very limited and long-term outcomes are lacking.

We present a case of ONJ identified in the United States, where a patient suffered an intermittent but long standing sore mouth related to exposed mandibular bone. 


\section{CASE SUMMARY}

A 66-year-old female presented with sore mouth ofduration of three weeks and exposed mandibular bone. The patient denied any history of obvious trauma to the affected area. Her medical history was significant for diabetes mellitus type1, hypertension, hypothyroidism, breast cancer, depression, osteoarthritis and osteopenia. She was taking aspirin, insulin, levothyroxine, bupropion, losartan, letrozole, atorvastatin and vitamin B12. Her breast cancer was treated by lumpectomy followed by chemotherapy. She denied history of breast cancer-related bisphosphonate therapy, however she was treated with once-yearly infusion of $5 \mathrm{mg}$ ZOL for osteopenia the last two consecutive years.

Intraoral examination revealed $8 \times 5 \mathrm{~mm}$ oval shaped ulcer with exposed bone and significant erythematous surround on the left posterior mandibular torus (Figures 1 and 2). There were no abnormal extraoral manifestations or radiographic findings noted. Based on clinical diagnosis of BRONJ and because the pain was occasional, management was palliative and no surgical intervention was rendered. The use of antibacterial oral rinse (0.12\% chlorohexidine gluconate) along with maintaining good oral hygiene and diabetes control (Patient reports consistent hemoglobin A1C of 9\%.) was advised for the patient. At the 2 weeks re-evaluation, the oral lesion was still present but pain-free. We recommended periodic follow-up at our clinic in addition to notifying her physician regarding her BRONJ.

One month later, our patient presented to the emergency clinic complaining of recurring severe pain and difficulty speaking associated with a tongue lesion. Intraorally, a mobile sequestra in the same area of the mandible and a traumatic ulceration on the adjacent tongue were noted. Healing mucosa and mild bleeding were visible underling the sequestra. At this time the sequestra

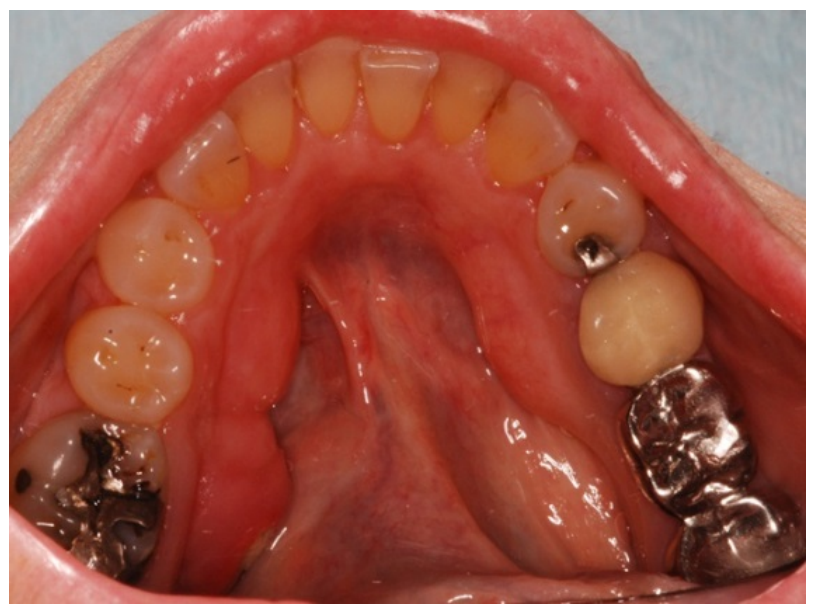

Figure 1. Occlusal view of the mandible showing exposed necrotic bone on the left posterior lingual tori.

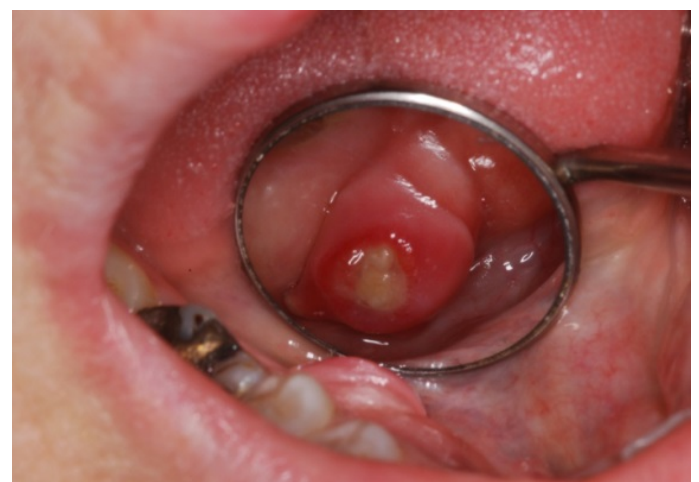

Figure 2. Mirror image showing exposed bone with inflammation in the surrounding area.

was removed under local anesthesia and the patient was instructed to continue using antibacterial oral rinse and follow-up. Complete healing of both lesions was observed in 3 weeks. The patient remains without signs and symptoms of recurrence at 12 months.

\section{DISCUSSION}

Clinical manifestations in our patient were typical for BRONJ. Dentoalveolar surgery such as tooth extraction is a common precipitating factor, yet, as seen in our case, up to $30 \%$ of BRONJ cases can occur spontaneously without known history of trauma to the affected area [5]. The mandible is affected more often than the maxilla, especially in the area of posterior torus covered by thin mucosa. Pain, swelling and fistula formation arecommon manifestations noted at the time of diagnosis. Jaw fracture may occur in severe cases. Radiographic finding such as diffused osteolytic lesions may not show unless in an advanced case, however, it is difficult todifferentiate from other chronic conditions such as osteomyelitis or osteoradionecrosis [6].

Our patient had multiple other known risk factors for developing ONJ: advancing age (older than 65 years), a history of malignancy, diabetes and chemotherapy $[7,8]$. IV administration of high potent NBPs particularly ZOL used in cancer patients is a major contributing factor for ONJ, however, our patient had no history of using such agents in that treatment regimen. Systemic conditions such as DM or chronic use of chemotherapeutic agents can decrease the inflammatory response and, as a result, increase the risk of ONJ. The negative impact of diabetes on oral health is well-established. Delayed healing and susceptible infection in patients with uncontrolled diabetes are always of concern for oral health care providers. The higher incidence of DM in patients with BRONJ compared with patients without BRONJ, potentially due to a compromised microvasculature and endothelial cell dysfunction, has been reported [9]. In our case, the patient's A1C was consistently around $9.0 \%$ during our 
observation. We believe that prolonged bone exposure was related to her diabetes condition.

It is noteworthy that our patient is taking Letrozole. Letrozole is an aromatase inhibitor used inthe treatment of hormonally-responsive breast cancer following surgery. Letrozole has antiandrogen effects and is a potential inhibitor of epithelial growth factor [10]. Although the exact etiology of BRONJ remains unclear, over suppression of bone turnover, inhibited angiogenesis and direct damage to oral mucosa have been considered to play important roles particularly with ZOL [11]. Letronidazole could further compromise the vasculature and oxygen perfusion in the jaw bone exacerbating the adverse effects of $\mathrm{ZOL}$.

Due to unpredictable healing outcomes in patients who developed BRONJ, the treatment is generally conservative. Invasive treatment such as surgical local debridement of the affected area is usually reserved for severe cases. Depending on the extent of infection, systemic antibiotics such as penicillin VK or amoxicillin and/or topical antibacterial oral suspension may be prescribed. Infection was localized in our patient case, therefore, the management was focused on maintenance of optimal oral hygiene and control of her diabetes. The concept of drug holidays in an attempt to reduce the risk of ONJ for patients planned for dento-alveolar surgery is controversial $[8,12]$. Our patient had the third infusion of $5 \mathrm{mg}$ ZOL three months after complete healing of her oral lesions. The comprehensive dental treatment plan was modified as a precautionary measure.

\section{CONCLUSION}

BRONJ as a disease process has multiple contributing risk factors. Although reported to be low, the incidence of BRONJ in osteopenic patients taking the once-yearly infusion of $5 \mathrm{mg}$ ZOL may be higher than previously thought, especially in those with multiple comorbidities. Coordinated efforts between physician and oral health care provider related to the risks and benefits of continuing antiresorptive therapy are imperative. All health care providers are encouraged to keep abreast of new developments in this field, assess the risk carefully and inform patients appropriately.

\section{REFERENCES}

[1] Marx, R.E. (2003) Pamidronate (aredia) and zoledronate (zometa) induced avascular necrosis of the jaws: A growing epidemic. Journal of Oral and Maxillofacial Surgery, 61, 1115-1117. http://dx.doi.org/10.1016/S0278-2391(03)00720-1

[2] Lo, J.C., O’Ryan, F.S., Gordon, N.P., et al. (2010) Prevalence of osteonecrosis of the jaw in patients with oral bisphosphonate exposure. Journal of Oral and Maxillofa- cial Surgery, 68, 243-253.

http://dx.doi.org/10.1016/j.joms.2009.03.050

[3] Boonen, S., Black, D.M., Colon-Emeric, C.S., et al. (2010) Efficacy and safety of a once-yearly intravenous zoledronic acid $5 \mathrm{mg}$ for fracture prevention in elderly postmenopausal women with osteoporosis aged 75 and older. Journal of the American Geriatrics Society, 58, 292-299. http://dx.doi.org/10.1111/j.1532-5415.2009.02673.x

[4] Grbic, J.T., Black, D.M., Lyles, K.W., et al. (2010) The incidence of osteonecrosis of the jaw in patients receiving 5 milligrams of zoledronic acid: Data from the health outcomes and reduced incidence with zoledronic acid once yearly clinical trials program. Journal of the American Dental Association, 141, 1365-1370.

[5] Woo, S.B., Hellstein, J.W. and Kalmar, J.R. (2006) Narrative [corrected] review: Bisphosphonates and osteonecrosis of the jaws. Annals of Internal Medicine, 144, 753761.

http://dx.doi.org/10.7326/0003-4819-144-10-200605160$\underline{00009}$

[6] Popovic, K.S. and Kocar, M. (2010) Imaging findings in bisphosphonate-induced osteonecrosis of the jaws. Radiology and Oncology, 44, 215-219.

[7] O’Ryan, F.S. and Lo, J.C. (2012) Bisphosphonate-related osteonecrosis of the jaw in patients with oral bisphosphonate exposure: Clinical course and outcomes. Journal of Oral and Maxillofacial Surgery, 70, 1844-1853. http://dx.doi.org/10.1016/j.joms.2011.08.033

[8] Hellstein, J.W., Adler, R.A., Edwards, B., et al. (2011) Managing the care of patients receiving antiresorptive therapy for prevention and treatment of osteoporosis: Executive summary of recommendations from the American dental association council on scientific affairs. Journal of the American Dental Association, 142, 1243-1251.

[9] Khamaisi, M., Regev, E., Yarom, N., et al. (2007) Possible association between diabetes and bisphosphonate-related jaw osteonecrosis. Journal of Clinical Endocrinology \& Metabolism, 92, 1172-1175.

http://dx.doi.org/10.1210/jc.2006-2036

[10] Wong, N.S., Buckman, R.A., Clemons, M., et al. (2010) Phase I/II trial of metronomic chemotherapy with daily dalteparin and cyclophosphamide, twice-weekly methotrexate, and daily prednisone as therapy for metastatic breast cancer using vascular endothelial growth factor and soluble vascular endothelial growth factor receptor levels as markers of response. Journal of Clinical Oncology, 28, 723-730.

http://dx.doi.org/10.1200/JCO.2009.24.0143

[11] Landesberg, R., Woo, V., Cremers, S., et al. (2011) Potential pathophysiological mechanisms in osteonecrosis of the jaw. Annals of the New York Academy of Sciences, 1218, 62-79. http://dx.doi.org/10.1111/j.1749-6632.2010.05835.x

[12] Yamashita, J. and McCauley, L.K. (2012) Antiresorptives and osteonecrosis of the jaw. Journal of Evidence-Based Dental Practice, 12, 233-247. http://dx.doi.org/10.1016/S1532-3382(12)70046-5 